\title{
Frecuencia de secuela renal pos evento agudo en síndrome urémico hemolítico
}

\author{
Frequency of renal sequela after acute uremic hemolytic syndrome
}

\author{
Jose Antonio Lulli-Cantoni ${ }^{1, a}$, Juan Miyahira ${ }^{1, b, 2, c}$
}

\section{RESUMEN}

Objetivos: Determinar la frecuencia de secuela renal después del evento agudo de Síndrome Urémico Hemolítico (SUH) en niños y los factores asociados a la presencia de secuelas. Material y métodos: Estudio descriptivo y retrospectivo. Se revisaron las historias clínicas de los pacientes con SUH en el HNCH de 1997 al 2012, y se registraron datos de características clínicas, hallazgos de laboratorio y parámetros de función renal al ingreso, al alta y a los seis meses después del alta. En los casos que no contaban con control a los 6 meses, se intentó contactar a los pacientes para tomar nuevos controles. Resultados: Siete de 12 pacientes presentaron disminución en la tasa de filtración glomerular (TFG) o proteinuria o hipertensión a los 6 meses o más después del episodio agudo, con un promedio de 30,75 meses de seguimiento. Tanto en los pacientes con secuelas como con recuperación renal se encontró una distribución similar de los factores asociados a secuelas como hipertensión al alta, proteinuria al alta, necesidad de diálisis, oligoanuria, leucocitosis $>20000 \mathrm{cel} / \mathrm{mm}^{3}$ y síntomas neurológicos. Sin embargo algunas variables como oligoanuria y necesidad de diálisis se encontraron a niveles por debajo de los descritos en la literatura. Conclusiones: Es claro que existen pacientes con secuelas renales luego del episodio agudo de SUH que además presentan varios factores predictores de secuelas descritos en la literatura.

PALABRAS CLAVE: Niños, enfermedad renal crónica, proteinuria, hipertensión, tasa de filtración glomerular, lesión renal aguda. (Fuente: DeCS BIREME).

\section{SUMMARY}

Objective: To determine the frequency of and associated factors to develop renal sequela after an acute episode of hemolytic uremic syndrome (HUS) in children. Methods: A descriptive-retrospective study that evaluated medical charts of patients with HUS at HNCH from 1997 to 2012 was conducted. Clinical features, laboratory findings including renal function tests on admission, discharge and after 6 months of follow-up were retrieved. Patients with incomplete data on renal function tests were contacted to complete the missing information. Results: 7 out of 12 patients presented a reduction in the glomerular filtration rate (GFR), proteinuria or hypertension 6 months or more after the acute episode, with a mean of 30,75 months of follow-up. Factors associated with sequela such as hypertension, proteinuria at discharge, need for dialysis, oligoanuria, leukocytosis $>20000 \mathrm{cells} / \mathrm{mm} 3$ and neurologic symptoms were equally distributed among patients with and without sequela. However, the frequency of proteinuria and oligoanuria were below previously reported rates. Conclusions: Renal sequela does occur after an acute episode of HUS with predicting factors well described in the medical literature.

KEYWORDS: Children, chronic kidney disease, proteinuria, hypertension, glomerular filtration rate, acute renal failure.

\footnotetext{
Facultad de Medicina Alberto Hurtado, Universidad Peruana Cayetano Heredia. Lima, Perú.

Servicio de Nefrología, Departamento de Medicina, Hospital Nacional Cayetano Heredia. Lima, Perú.

Egresado; ${ }^{b}$ Profesor Principal; ${ }^{c}$ Médico Cirujano, especialista en Nefrología.
} 


\section{INTRODUCCIÓN}

El Síndrome Urémico Hemolítico (SUH) es una enfermedad caracterizada por la triada de anemia hemolítica microangiopática, trombocitopenia e insuficiencia renal aguda. Se clasifica de acuerdo a la presencia o no de pródromo diarreico, siendo el cuadro precedido por diarrea causado por la infección de bacterias productoras de verotoxina y ocurre principalmente en niños menores de 5 años (1).

En Argentina y Uruguay la incidencia de SUH llega hasta 10,5 casos/100 000 habitantes por año y es la primera causa de insuficiencia renal aguda (IRA) y entre las primeras causas de enfermedad renal crónica (ERC) en población pediátrica $(2,3)$.

En la literatura se han descrito varios factores asociados con mal pronóstico en SUH; los síntomas neurológicos, duración de oliguria y diálisis, hipertensión, entre otros, han mostrado asociación de diferente grado con la presencia de secuelas renales en varios estudios. Sin embargo otros como edad, sexo, el grado de anemia, trombocitopenia, entre otros tienen resultados inconsistentes (4).

En el Perú se han realizado estudios que describen las características clínicas y epidemiológicas de SUH más no el pronóstico $(5,6)$. Garg AX y col (4), en una revisión sistemática y metanálisis encuentran entre 0 y $30 \%$ de mortalidad o enfermedad renal crónica terminal (ERCT) y entre $0-64 \%$ de otras secuelas renales.

La variabilidad en la frecuencia de ocurrencia de las secuelas sumadas a la falta de factores predictores sólidos y la incertidumbre del tiempo adecuado para el seguimiento hacen que el pronóstico de la mayoría de pacientes con SUH sea incierto. Es importante conocer la evolución posterior al cuadro agudo y la frecuencia de los posibles factores pronósticos para tener mayor información para el manejo posterior de estos pacientes.

Por estas razones y por la falta de estudios nacionales se realizó el estudio cuyo objetivo fue determinar la frecuencia de secuelas renales después del evento agudo en niños con SUH y los factores asociados a la presencia de secuelas.

\section{MATERIAL Y MÉTODOS}

Se realizó un estudio descriptivo retrospectivo, tipo reporte de casos. Se revisó el libro de registro de atenciones de nefrología pediátrica del Hospital Nacional Cayetano Heredia para identificar a los pacientes con diagnóstico de Síndrome Urémico Hemolítico entre el 1 de enero de 1997 y el 31 de diciembre de 2012; luego se revisaron las historias clínicas de los pacientes. Se incluyeron a todos los pacientes menores de 14 años con datos completos referentes al estudio.

Los criterios de inclusión fueron: Diagnóstico de síndrome urémico hemolítico, edad menor de 14 años de ambos sexos y tener historia clínica con datos de seguimiento de 6 o más meses. Los criterios de exclusión fueron: tener historia clínica con datos incompletos o no contar con historia clínica.

Se registraron parámetros clínicos y de laboratorio en tres momentos, al ingreso: edad, sexo, duración de hospitalización, volumen urinario, proteinuria, tensión arterial, creatinina sérica, tasa de filtración glomerular (TFG) calculada, examen de orina, hematocrito y hemoglobina sérica; durante la hospitalización: número de días en diálisis, número de días en oligoanuria $(<1$ $\mathrm{ml} / \mathrm{kg} /$ hora en niños o $<0,5 \mathrm{ml} / \mathrm{kg} /$ hora en lactantes) y manifestaciones extra renales asociadas: elevación de leucocitos mayor a $20000 / \mathrm{mm}^{3}$ a predominio de neutrófilos, disminución de nivel de conciencia, accidente cerebrovascular, convulsiones y colitis isquémica; al alta: creatinina sérica, TFG calculada, proteinuria, volumen urinario, tensión arterial, examen de orina, hematocrito, hemoglobina sérica, y a los 6 o más meses de seguimiento luego del evento agudo: tiempo luego del ingreso, creatinina sérica, TFG calculada, tensión arterial, proteinuria, volumen urinario, examen de orina, hematocrito y hemoglobina sérica. El cálculo de la TFG se realizó con la ecuación de Schwartz (7).

Seis pacientes no tuvieron controles luego de los seis meses del alta. Se intentó ubicar a estos pacientes por medio telefónico a través del Departamento de Servicio Social del hospital. Se logró ubicar a cuatro pacientes, que fueron citados en el consultorio de nefrología pediátrica donde se conversó con los padres del menor y se pidió firmar un consentimiento o asentimiento informado según correspondía y se les realizó controles de depuración de creatinina, proteinuria en 24 horas, hemoglobina, hematocrito, examen completo de orina y toma de tensión arterial.

Para el diagnóstico de SUH se consideró la presencia de anemia hemolítica microangiopática 
(hemoglobina menor a $12 \mathrm{~g} / \mathrm{dl}$ con presencia de esquistocitos en el estudio de lámina periférica), insuficiencia renal aguda (creatinina sérica mayor a $1,0 \mathrm{mg} / \mathrm{dl}$ ) y trombocitopenia (plaquetas menor a $150000 / \mathrm{mm}^{3}$ ) además de la presencia de pródromo diarreico. Un paciente presentó hemoglobina de $12 \mathrm{~g} /$ $\mathrm{dl}$ al ingreso y otro creatinina sérica de $0,87 \mathrm{mg} / \mathrm{dl}$ al ingreso, pero por presentar el resto de características del cuadro clínico y una evolución coherente con el diagnóstico se les considero con SUH. El resto de pacientes cumplieron los criterios de SUH.

Se consideró presencia de secuela renal si el paciente presentaba TFG estimada $<80 \mathrm{ml} / \mathrm{min} / 1,73 \mathrm{~m}^{2}$ o hipertensión arterial o proteinuria después de seis meses del diagnóstico y recuperación renal si no presentaba hipertensión ni proteinuria y la TFG estimada era $>90 \mathrm{ml} / \mathrm{min} / 1,73 \mathrm{~m}^{2}$ al alta o a los seis meses luego del diagnóstico. Se consideró hipertensión arterial con una medida de tensión arterial sistólica o diastólica sobre el percentil 95 de la escala de la CDC respecto a su talla y edad (8).

Al inicio del estudio se planeó considerar proteinuria si era mayor de $4 \mathrm{mg} / \mathrm{m}^{2} /$ hora en una colección de 12 ó 24 horas, sin embargo, en la revisión de las historia clínicas solo se encontraron valores de proteinuria cualitativas y semi-cuantitativas al ingreso y al alta, por lo cual se consideró proteinuria si era mayor o igual a $30 \mathrm{mg} / \mathrm{dl}$, en la tira reactiva. En el seguimiento se encontró que la proteinuria fue evaluada con la razón proteinuria/creatinina en orina, por ello, se utilizó $0,5 \mathrm{~g}$ de proteinuria sobre gramo de creatinina, como punto de corte en lactantes, para definir presencia de proteinuria. Se consideró hematuria a la presencia de
5 o más hematíes por campo de alto poder $(400 \mathrm{X})$ en orina.

Se generó una ficha de recolección de datos en la que se registraron los datos de la revisión de las historias; luego se ingresaron los datos en una hoja de cálculo en Microsoft Excel ${ }^{\circledR}$ previa codificación para salvaguardar la información personal de los pacientes. Se utilizó estadística descriptiva, media y desviación estándar o rango para las variables continuas y tabla de frecuencias para las variables discretas. Se comparó el grupo con secuelas y sin ellas respecto a la frecuencia de factores pronósticos al inicio, durante y al finalizar la hospitalización. Se utilizó la prueba t pareada para evaluar si existía diferencia entre las medias de algunas variables en dos momentos, inicio y alta y alta y último control.

El estudio fue revisado y aprobado por el Comité Institucional de Ética de la Universidad Peruana Cayetano Heredia y el Comité Institucional de Ética en la Investigación del Hospital Nacional Cayetano Heredia.

\section{RESULTADOS}

En el libro de registro de atenciones de Nefrología Pediátrica se encontraron 33 pacientes con diagnóstico de SUH, en 15 de ellos las historias clínicas se encontraban en el archivo pasivo y no se pudo disponer de ellas, siendo excluidos del estudio. Dos pacientes fueron diagnosticados en otro hospital y sus historias no tenían datos completos; un paciente falleció durante su hospitalización. Un paciente falleció antes sin haber acudido a su primer control luego del alta y no se logró

Tabla 1. Características clínicas en los pacientes al ingreso y al alta del episodio agudo de SUH (n=12).

\begin{tabular}{|c|c|c|c|c|c|c|c|}
\hline \multirow{2}{*}{ Características clínicas } & \multicolumn{2}{|c|}{ Ingreso } & \multirow{2}{*}{$\frac{\text { Alta }}{\text { Media } \pm \mathrm{DE}^{*}}$} & \multicolumn{4}{|c|}{ t-Test pareado } \\
\hline & Media $\pm \mathrm{DE}^{*}$ & Rango & & Rango & Diferencia & IC* $95 \%$ & $\mathbf{p}$ \\
\hline Tensión arterial sistólica & $110.83 \pm 12,35$ & $90-130$ & $101,67 \pm 12,80$ & $80-130$ & 9,17 & $-1,02-19,36$ & 0,073 \\
\hline $\begin{array}{l}\text { Tensión arterial } \\
\text { diastólica }\end{array}$ & $63,75 \pm 7,39$ & $50-80$ & $57,50 \pm 8,29$ & $50-80$ & 6,25 & $-0,54-13,03$ & 0,068 \\
\hline Tensión arterial media & $79,44 \pm 8,71$ & $63,33-96,67$ & $72,22 \pm 8,75$ & $63,33-96,67$ & 7,22 & $0,37-14,08$ & 0,040 \\
\hline Flujo urinario $(\mathrm{ml} / \mathrm{kg} / \mathrm{h})$ & $0,67 \pm 0,97$ & $0-3,23$ & $2 \pm 0.77$ & $0,93-3,10$ & 1,26 & $0,49-2,02$ & 0,004 \\
\hline Creatinina sérica (mg/dl) & $3,78 \pm 2,49$ & $0,87-10,5$ & $1 \pm 0,51$ & $0,3-2,2$ & 2,76 & $1,13-4,39$ & 0,003 \\
\hline $\mathrm{TFG}\left(\mathrm{ml} / \mathrm{min} / 1.73 \mathrm{~m}^{2}\right)$ & $18,21 \pm 13,01$ & $4-47,4$ & $57,03 \pm 35,21$ & $18-154$ & 38,82 & $19,74-57,89$ & 0,000 \\
\hline Proteinuria $(\mathrm{mg} / \mathrm{dl}) \dagger$ & $208,57 \pm 206,08$ & $0-500$ & $141,32 \pm 266,71$ & $0-791$ & 67,25 & $-163,99-298,49$ & 0,503 \\
\hline Hematocrito (\%) & $22,18 \pm 5,93$ & $14-34$ & $24,92 \pm 4,03$ & $19-33$ & 2,73 & $-2,51-7,98$ & 0,227 \\
\hline
\end{tabular}

*: DE, Desviación estándar. IC, Intervalo de confianza

$\dagger$ : Análisis de t-Test para proteinuria para 7 pacientes por falta de datos pareados 
contactar a los padres para conocer la causa de muerte. Además dos pacientes no volvieron al hospital para sus controles, y tampoco pudieron ser contactados. Al final doce pacientes fueron incluidos en el estudio.

Cinco $(41,67 \%)$ fueron de sexo femenino, la edad promedio fue 17,83 meses (rango de 11 a 27 meses) al momento del diagnóstico. Todos los pacientes presentaron disminución de la tasa de filtración glomerular de algún grado y la mayoría hipertensión tanto como proteinuria. Dos pacientes presentaron anuria por lo cual no se pudo determinar la presencia de proteinuria ni hematuria al ingreso. Todos los pacientes presentaron por lo menos dos marcadores de enfermedad renal, como era esperado. Diez (83.33\%) pacientes presentaron hipertensión y disminución de la TFG y de ellos 4 presentaron además proteinuria y hematuria (Tablas 1 y 2).

Tabla 2. Marcadores de enfermedad renal en los pacientes al ingreso y al alta del episodio agudo de SUH ( $\mathrm{n}=12)$.

\begin{tabular}{lcccc}
\hline \multirow{2}{*}{ Marcadores de enfermedad renal* } & \multicolumn{2}{c}{ Ingreso } & \multicolumn{3}{c}{ Alta } \\
\cline { 2 - 5 } & $\mathbf{n}+$ & $\mathbf{\%}$ & $\mathbf{n}+$ & $\mathbf{\%}$ \\
\hline Solo Proteinuria & 0 & 0 & $1 / 10$ & 10,0 \\
Solo Disminución de la TFG & 0 & 0 & $2 / 12$ & 16,7 \\
Proteinuria + Disminución de la TFG & $1 / 8$ & 12,5 & $1 / 10$ & 10,0 \\
Hipertensión + Disminución de la TFG & $6 / 12$ & 50,0 & $1 / 12$ & 8,3 \\
Disminución de la TFG + Hematuria & 0 & 0 & $2 / 10$ & 20,0 \\
Proteinuria + Hipertensión + Disminución de la TFG & 0 & 0 & $1 / 10$ & 10,0 \\
Proteinuria + Hipertensión + Hematuria & 0 & 0 & $1 / 10$ & 10,0 \\
Proteinuria + Disminución de la TFG + Hematuria & $1 / 8$ & 12,5 & $2 / 10$ & 20,0 \\
Proteinuria + Hipertensión + Disminución de la TFG + Hematuria & $4 / 8$ & 50,0 & $1 / 10$ & 20,0 \\
\hline
\end{tabular}

*: Proteinuria: $>30 \mathrm{mg} / \mathrm{dl}$, Disminución de la TFG: $<80 \mathrm{ml} / \mathrm{min} / 1.73 \mathrm{~m}^{2}$, Hematuria: $>5$ hematíes por campo de alto poder. No hubo pacientes con solo hematuria al ingreso o alta

$\dagger$ : Dos pacientes tuvieron anuria al ingreso y dos no tuvieron registro de muestra de orina, no se tiene información sobre proteinuria o hematuria en cuatro pacientes.

t: Dos pacientes no tienen registro de proteinuria ni hematuria al alta.

Tabla 3. Características clínicas en los pacientes al alta y en el seguimiento $(\mathrm{n}=12 *)$.

\begin{tabular}{|c|c|c|c|c|c|c|c|}
\hline \multirow{2}{*}{ Características clínicas } & \multicolumn{2}{|c|}{ Alta } & \multicolumn{2}{|c|}{ Seguimiento } & \multicolumn{3}{|c|}{ t-Test pareado } \\
\hline & Media $\pm \mathrm{DE} \dagger$ & Rango & Media $\pm \mathrm{DE} \dagger$ & Rango & Diferencia & $\mathrm{IC}+95 \%$ & $\mathbf{p}$ \\
\hline Tensión arterial sistólica & $101,67 \pm 12,80$ & $80-130$ & $98,75 \pm 7,11$ & $80-110$ & 2,92 & $-5,04-10,87$ & 0,437 \\
\hline $\begin{array}{l}\text { Tensión arterial } \\
\text { diastólica }\end{array}$ & $57,50 \pm 8,29$ & $50-80$ & $61,67 \pm 3,12$ & $60-70$ & 4,17 & $-1,39-9,72$ & 0,127 \\
\hline Tensión arterial media & $72,22 \pm 8,75$ & $63,33-96,67$ & $74,03 \pm 3,57$ & $66,67-80$ & 1,81 & $-3,64-7,25$ & 0,481 \\
\hline Creatinina sérica (mg/dl) & $1 \pm 0,51$ & $0,3-2,2$ & $0,49 \pm 0,15$ & $0,3-0,85$ & 0,52 & $0,15-0,89$ & 0,009 \\
\hline $\mathrm{TFG}\left(\mathrm{ml} / \mathrm{min} / 1,73 \mathrm{~m}^{2}\right)$ & $57,03 \pm 35,21$ & $18-154$ & $107,97 \pm 27,67$ & $57-176$ & 50,94 & $19,48-82,40$ & 0,004 \\
\hline Proteinuria & $141,32 \pm 266,71$ & $0-791$ & $0,34 \pm 0,24$ & $0-0,79$ & --- & --- & --- \\
\hline Hematocrito (\%) & $24,92 \pm 4,03$ & $19-33$ & $36,98 \pm 2,58$ & $32-42,9$ & 12,06 & $9,01-15,11$ & 0,000 \\
\hline
\end{tabular}

*: Seguimiento mayor a 2 meses, promedio $42.75 \pm 48.98$ meses.

$\uparrow$ : DE: Desviación Estándar. IC: Intervalo de confianza.

†: Datos de proteinuria al alta presentados en $\mathrm{mg} / \mathrm{dl}$ y en el seguimiento en indice de proteinuria/creatinina. No se pudo realizar t-Test. Dos pacientes se encontraban tomando Enalapril por proteinuria diagnosticada en anteriores controles luego del episodio de SUH. Se consideraron con proteinuria para la descripción de secuelas renales. 
El tiempo de hospitalización promedio fue 15,33 \pm 6,61 días (rango 7-28) y presentaron en promedio 5,58 $\pm 4,27$ días de oliguria (rango $0-11$ ). Nueve de los 12 pacientes requirieron diálisis peritoneal y estuvieron en diálisis en promedio 7,92 $\pm 5,36$ días (rango $5-15$ días). En relación a las manifestaciones extra renales asociadas a mal pronóstico, cinco pacientes presentaron leucocitosis a predominio de neutrófilos sobre 20000 células $/ \mathrm{mm}^{3}$; uno presentó disminución del nivel de conciencia transitorio, cinco presentaron convulsiones tónico clónicas y un paciente tuvo accidente cerebro vascular (infarto cerebral izquierdo). Ningún paciente presentó colitis isquémica.
Al alta, los valores promedio de tensión arterial sistólica, diastólica y media en promedio disminuyeron, pero solo la disminución de la presión arterial media alcanzó significación estadística $(\mathrm{p}=0,040)$. La proteinuria disminuyó en $67,25 \mathrm{mg} / \mathrm{dl}$ en promedio, pero no alcanzó a tener significación estadística. El flujo urinario aumentó en promedio $1,26 \mathrm{ml} / \mathrm{kg} / \mathrm{h}$ y alcanzó significación estadística $(\mathrm{p}=0,004)$. La TFG mejoró y la creatinina sérica disminuyó de manera significativa $(\mathrm{p}=0,000$ y $\mathrm{p}=0,003$ respectivamente). El hematocrito aumentó discretamente en promedio, pero no alcanzó diferencia significativa. Al momento del alta todos los pacientes aún presentaban uno o más

Tabla 4. Frecuencias de factores asociados a secuelas renales en SUH en pacientes con y sin marcadores de enfermedad renal $(n=12)$.

\begin{tabular}{|c|c|c|}
\hline Factor asociado & $\begin{array}{c}\text { Pacientes con secuela } \\
\text { renal }(n=7)\end{array}$ & $\begin{array}{c}\text { Pacientes sin secuela } \\
\text { renal }(n=5)\end{array}$ \\
\hline \multicolumn{3}{|l|}{ Tiempo de hospitalización } \\
\hline$<14$ días & 4 & 2 \\
\hline$\geq 14$ días & 3 & 3 \\
\hline Proteinuria al ingreso* & $4 / 5$ & $2 / 3$ \\
\hline Hipertensión arterial al ingreso & 6 & 4 \\
\hline Creatinina sérica $>2 \mathrm{mg} / \mathrm{dl}$ al ingreso & 5 & 4 \\
\hline $\mathrm{TFG}<18 \mathrm{ml} / \mathrm{min} / 1,73 \mathrm{~m}^{2}$ al ingreso & 4 & 4 \\
\hline \multicolumn{3}{|l|}{ Oligoanuria } \\
\hline$<4$ días & 3 & 2 \\
\hline$\geq 4$ días & 4 & 3 \\
\hline Necesidad de diálisis & 5 & 4 \\
\hline$<7$ días & $2 / 5$ & $1 / 4$ \\
\hline$\geq 7$ días & $3 / 5$ & $3 / 4$ \\
\hline Leucocitosis $>20000 / \mathrm{mm}^{3}$ & 3 & 2 \\
\hline Convulsiones & 2 & 3 \\
\hline Disminución de nivel de conciencia & 1 & 0 \\
\hline Accidente cerebro vascular & 1 & 0 \\
\hline Proteinuria al alta $\dagger$ & $5 / 6$ & $2 / 4$ \\
\hline Hipertensión al alta & 1 & 3 \\
\hline Creatinina sérica $>1 \mathrm{mg} / \mathrm{dl}$ al alta & 1 & 2 \\
\hline $\mathrm{TFG}<80 \mathrm{ml} / \mathrm{min} / 1,73$ al alta & 6 & 4 \\
\hline
\end{tabular}




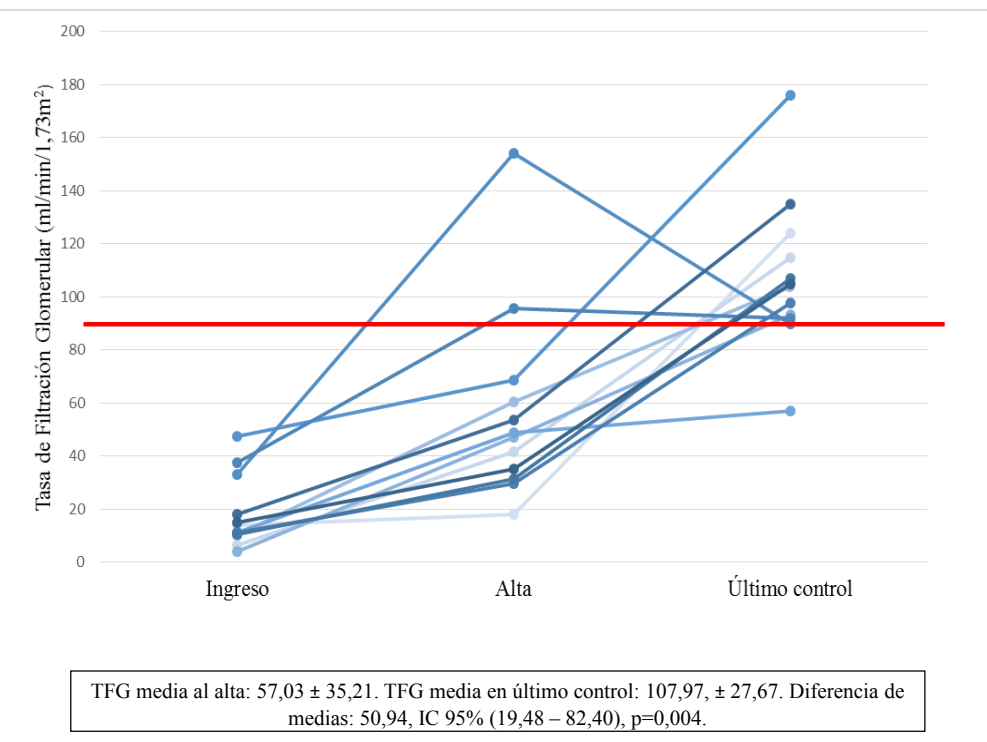

Gráfico 1. Evolución de la Tasa de filtración glomerular: al ingreso, al alta y en el último control (n=12).

marcadores de enfermedad renal: 10/12 presentaron disminución de la TFG, 4/12 hipertensión, 7/12 proteinuria y 6/12 hematuria (Tablas 1 y 2 ).

Luego de 6 meses de seguimiento 7/12 (58\%) pacientes presentaron un marcador de enfermedad renal, a pesar de observar promedios dentro de los parámetros normales para tensión arterial, creatinina sérica, TFG y proteinuria; 3/7 presentaron solo proteinuria $(>0,5 \mathrm{~g} / \mathrm{g}$ de proteinuria/creatinina en orina), 3/7 solo hipertensión y 1/7 solo disminución de la TFG $\left(<80 \mathrm{ml} / \mathrm{min} / 1,73 \mathrm{~m}^{2}\right) .5 / 12$ pacientes no presentaron algún marcador de enfermedad renal. Dos pacientes se encontraban en tratamiento con enalapril. El tiempo promedio hasta el control fue 42,75 \pm 48,98 meses (rango 2 - 156 meses). Tanto la tensión arterial sistólica, diastólica y media no presentaron diferencias significativas, La TFG aumentó en 50,94 $\mathrm{ml} / \mathrm{min} / 1,73 \mathrm{~m}^{2}$ y la creatinina sérica disminuyó en $0,52 \mathrm{mg} / \mathrm{ml}$, ambos estadísticamente significativos al igual que el aumento de hematocrito en $12,06 \%$ ( $\mathrm{p}=$ $0,009, \mathrm{p}=0,004$ y $\mathrm{p}=0,000$ respectivamente) (Tabla $3)$.

El gráfico 1, muestra la evolución de la TFG de los pacientes; se puede apreciar que en todos los pacientes con controles después del alta la TFG mejoró en grado variable. Solo un paciente presentó TFG menor de $80 \mathrm{ml} / \mathrm{min} / 1,73 \mathrm{~m} 2$ en su último control, y fue en un control luego de los seis meses de seguimiento por ello en este caso se consideró la disminución de TFG como secuela. Los promedios del alta y el último control (mayor a 4 meses) presentaron diferencia significativa $(\mathrm{p}=0,004)$.
En la tabla 4, se muestra la frecuencia de los factores asociados a secuela renal en el grupo con secuela renal y sin ella. Se observó que el grupo con secuela renal presentó un mayor número de pacientes con proteinuria al alta, mientras que el grupo sin secuelas presentó más pacientes con hipertensión al alta. El resto de los factores asociados encontraron una distribución similar en ambos grupos.

\section{DISCUSIÓN}

Se encontró $7 / 12 \quad(58,3 \%)$ pacientes que desarrollaron secuelas renales luego del evento agudo de SUH, manifestada como disminución de la TFG, hipertensión o proteinuria. El seguimiento de los pacientes en nuestro estudio fue en promedio 3,5 años (42,75 meses) después del episodio de SUH y si bien no encontramos a algún paciente con enfermedad renal crónica terminal (ERCT) o fallecidos por causa de enfermedad renal, se encontró presencia de marcadores que los ponen en riesgo de desarrollarla posteriormente. Se ha reportado entre el $25 \%$ al $51 \%$ de secuelas en niños con SUH luego de un seguimiento promedio de 4 a 6,5 años $(4,9)$. Otro estudio que evaluó las secuelas en episodios agudos de diferentes causas de IRA reportaron secuelas en el 46\% luego de un seguimiento de 1 a 3 años (10).

Varios estudios han reportado a la disminución de la TFG como la secuela más frecuente (4). En nuestro estudio las secuelas observadas fueron: 3 casos de hipertensión, 3 casos de proteinuria y un caso de diminución de TFG. 
Se han propuesto algunos factores como predictores del pronóstico renal luego del episodio agudo de $\mathrm{SUH}$; sin embargo, varios aún están en estudio para determinar su asociación con el pronóstico (4). En nuestro estudio, de manera general, los factores propuestos estuvieron distribuidos tanto en el grupo con y sin secuela renal, salvo la presencia de proteinuria al alta en el grupo con secuela y la presencia de hipertensión al alta en el grupo sin secuela renal.

Respecto a presencia de oliguria se ha reportado un peor pronóstico en pacientes con más de 4 días de oliguria (11), algunos estudios muestran una mayor frecuencia de secuelas luego de los 8 a 10 días de oliguria $(4,12)$. Cabe mencionar que en nuestro estudio, la proporción de pacientes con secuela y sin secuela que presentaron oliguria mayor a 4 días fue similar y también fue similar la proporción de pacientes con oliguria mayor a 8 días en ambos grupos (2/7 y 1/5, respectivamente). Además, un paciente de cada grupo presentó 24 horas de anuria, lo cual también ha sido reportado como un marcador de mal pronóstico (13).

La necesidad de diálisis mayor de 4 semanas u hospitalización mayor de 3 semanas $(4,14)$ han sido propuestas como factores asociados a secuelas renales. En nuestro estudio tanto el tiempo de necesidad de diálisis como el tiempo de hospitalización se encuentran por debajo de estos valores. Solo 2 pacientes con secuela presentaron un tiempo de hospitalización mayor de 21 días. Ninguno de los pacientes con o sin secuela presentó un periodo de necesidad de diálisis mayor de 15 días.

Otros factores de pronóstico asociados como leucocitosis mayor de $20 \quad 000 / \mathrm{mm}^{3}$ y síntomas neurológicos como convulsiones, disminución del nivel de conciencia y accidente cerebro vascular $(4,15)$, tuvieron una distribución similar en ambos grupos.

Hipertensión es un factor asociado a mal pronóstico $(4,14)$. La proporción de pacientes con hipertensión arterial al ingreso fue similar en ambos grupos, y al alta inclusive la proporción de hipertensos fue más alta en el grupo sin secuela renal.

También, tanto la proteinuria como la micro albuminuria han sido descritos como factores asociados $(13,14,16)$, en nuestro estudio la proporción de pacientes con proteinuria al ingreso fue similar en ambos grupos, pero el grupo con secuela renal tuvo una mayor cantidad de casos de proteinuria al alta que el grupo sin secuela. Hubo un caso que desarrolló proteinuria en el seguimiento.

Entre las limitaciones del estudio debemos mencionar número pequeño de casos y el número de casos excluidos por no contar con la fuente de información. Además la falta de uniformidad en la medición de proteinuria y el hecho que algunos análisis no detecten micro albuminuria (16). Asimismo, hay que tener en consideración que el Hospital Nacional Cayetano Heredia es un hospital de referencia y debido a ello los casos incluidos en el estudio puedan corresponder a casos más complejos y por lo tanto con un mayor riesgo de presentar secuelas.

En el registro que revisamos figuran 15 casos de SUH a cuyas historias clínicas no se tuvo acceso. No podemos asegurar que estos pacientes excluidos tengan la misma distribución de secuelas renales que los pacientes incluidos en el estudio, ni la distribución de los factores de riesgo asociados al desarrollo de secuela renal. Algunos de estos pacientes podrían haber presentado secuela renal y estar en seguimiento en los hospitales del seguro social (EsSALUD) o algún establecimiento privado.

Consideramos que observando la presencia de secuelas en los pacientes con SUH, se recomienda un seguimiento prolongado en pacientes que presentan secuelas luego de los 6 meses y valorar la necesidad de realizar controles en los pacientes sin secuelas. La información descrita en otros estudios $(17,18)$ sobre la disminución de la frecuencia de secuelas en brotes de SUH localizados y los síntomas de la infección por E. coli y otros causales de esta enfermedad plantearía que depende de la cepa que genere SUH podríamos obtener diferente número de secuelas. Ishikura y col (19), han descrito a una cohorte de niños ERC estadio 3 a 5 donde el 12,5\% progresaron a ERCT en 1,49 años. Por ello recomendamos estudios con no solo con un mayor número de pacientes, sino con seguimientos más prolongados para intentar detectar a los niños con marcadores de enfermedad renal leves que pudieran progresar rápidamente. Además sería ideal identificar las cepas que causaron la enfermedad y si tienen relación con las secuelas.

A pesar de las limitaciones de este estudio, es claro que existen pacientes que tienen secuelas renales luego de un episodio de SUH. No tenemos herramientas sólidas para predecir que pacientes tendrán secuelas luego, pero debemos continuar evaluando los marcadores de enfermedad renal por encima de los 2 
años luego del episodio agudo hasta tener estudios con evidencia más sólida sobre el pronóstico y el tiempo de seguimiento en nuestro propio medio.

\section{Declaración de financiamiento y de conflictos de intereses:}

El estudio fue financiado por JALC, los autores declaran no tener conflictos de intereses.

\section{Contribución de autoría:}

JALC participó en el diseño del estudio, recolección de los datos, interpretación de los resultados y redacción del artículo. JM participó en el diseño del estudio, interpretación de los datos, revisión crítica del artículo.

\section{Correspondencia:}

José Antonio Lulli

Calle Paracas 180 Urbanización El sol de la Molina

Lima 12, Perú.

Correo electrónico: jose.lulli@upch.pe

\section{REFERENCIAS BIBLIOGRÁFICAS}

1. Mele C, Remuzzi G, Noris M. Hemolytic uremic Syndrome. Semin Immunopathol. 2014; 36(4):399420.

2. Noris M, Remuzzi G. Hemolytic uremic syndrome. J Am Soc Nephrol. 2005; 16(4):1035-50.

3. Rivas M, Caletti MG, Chinen I, et al. Home-prepared hamburger and sporadic hemolytic uremic syndrome Argentina. Emerg Infect Dis. 2003; 9(9):1184-1186.

4. Garg AX. Suri RS, Barrowman N, et al. Long-term Renal Prognosis of Diarrhea-Associated Hemolytic Uremic Syndrome: A systematic Review, Meta-analysis and Meta-regression. JAMA. 2003; 290(10):1360-1370.

5. Castillo W. Síndrome urémico hemolítico en niños. Tesis para optar el grado de Bachiller en Medicina. Lima, Perú: Universidad Peruana Cayetano Heredia, 1989. $43 \mathrm{pp}$.

6. Sakihara GPB, Reyna R, Mendoza A, et al. Aspectos clínicos y terapéuticos del Síndrome urémico hemolítico. Revista Peruana de Pediatría. 2001; 54(2):8-13.

7. Schwartz GJ, Brion LP, Spitzer A, et al. The use of plasma creatinine concentration for estimating glomerular filtration rate in infants, children, and adolescents. Pediatr Clin North Am. 1987; 34(3):57190.
8. The fourth report on the diagnosis, evaluation, and treatment of high blood pressure in children and adolescents. Pediatrics. 2004;114(S2):555-76.

9. Siegler RL, Pavia AT, Christofferson RD, et al. A 20Year Population-Based study of postdiarrheal hemolytic uremic síndrome. Pediatrics. 1994; 94(1):35-40.

10. Mammen C, Abbas A, Skippen P, et al. Long-term Risk of CKD in children surviving episodes of acute kidney injury in the intensive care unit: A prospective cohort study. Am J Kidney Dis. 2012; 59(4):523-530.

11. Balestracci A, Martin SM, Toledo I, et al. Capacidad del período oligoanúrico para predecir secuela renal en niños con síndrome urémico hemolítico asociado a diarrea. Arch Argent Pediatr. 2012; 110(3):221-226.

12. Oakes, RS, Kirkhamm JK, Nelson RD, et al. Duration of oliguria and anuria as predictors of chronic renal-r elated sequelae in post-diarrheal hemolytic uremic syndrome. Pediatr Nephrol. 2008; 23:1303-1308.

13. Spizzirri FD, Rahman RC, Bibiloni N, et al. Childhood hemolytic uremic syndrome in Argentina: long-term follow-up and prognostic features. Pediatr Nephrol. 1997; 11:156-160.

14. Fitzpatrick MM, Shah V, Trompeter RS, et al. Long term renal outcome haemolytic uraemic syndrome. BMJ. 1991; 303:489-492.

15. Rosales A, Hofer J, Zimmerhackl LB, et al. Need for long term follow-up in enterohemorrhagic Escherichia coli-associated hemolytic uremic syndrome due to late-emerging squelae. Clin Infect Dis. 2012; 54(10):1413-21.

16. Lou-Meda R, Oakes RS, Gilstrap, et al. Prognostic significance of microalbuminuria in post diarrheal hemolytic uremic syndrome. Pediatr Nephrol. 2007; 22:117-120.

17. Garg AX, Salvadori M, Okell JM, et al. Albuminuria and estimated GFR 5 years after Escherichia coli O157 hemolytic uremic syndrome: An Update. Am J Kidney Dis. 2008; 51:435-444.

18. Garg AX, Clark WF, Salvadori M. Absence of renal sequelae after childhood Escherichia coli O157:H7 gastroenteritis. Kidney Int. 2006; 70(4):807-12.

19. Ishikura K, Uemura O, Hamasaki Y, et al. Progression to end-stage kidney disease in Japanese children with chronic kidney disease: results of a nationwide prospective cohort study. Nephrol Dial Transplant. 2014; 29:878-884.

Recibido: 16/02/2015

Aceptado: 30/03/2015 